\title{
Simulação de Arquiteturas de Sistemas de Monitoramento de Quedas de Pacientes Domiciliares
}

\author{
Jorge T. Bruno, Renato F. Bulcão-Neto \\ Instituto de Informática - Universidade Federal de Goiás (UFG) \\ Caixa Postal 131 - 74.001-970 - Goiânia - GO- Brazil \\ jorgeteixeira@inf.ufg.br, rbulcao@ufg.br
}

\begin{abstract}
Falls are the most common cause of injuries for older adults. IoT systems have been developed for monitoring, detecting, and reporting falls of patients in the home environment. However, studies have reported that most falls occur in the bathroom, where older adults do not use wearable devices. A solution with sensors installed on the bathroom environment could contribute to increasing the degree of fall detection. The purpose of this paper is to show how simulation models can be used to evaluate, at design time, two architectural alternatives for IoT-based home monitoring systems. The first alternative is a conventional IoT system based on wearable devices, while the second architectural design improves the former model by including sensors on the bathroom environment. Datasets for fall detection were used during the simulations. Preliminary results show that fall detection increases of up to $8.2 \%$ by comparing an architecture that uses only accelerometers to one that additionally uses a doppler radar installed on the bathroom floor.
\end{abstract}

Resumo. Quedas são uma causa recorrente de hospitalização de idosos. Sistemas IoT têm sido desenvolvidos para monitorar, detectar e notificar quedas de pacientes no ambiente domiciliar. Entretanto, estudos têm reportado que a maioria das quedas ocorre no banheiro, local onde os idosos comumente não estão utilizados os dispositivos vestíveis que os monitoram para notificar serviços de saúde e resgate em caso de queda. Neste sentido, o uso de sensores instalados no ambiente do banheiro poderia contribuir para aumentar o grau de detecção de quedas. O objetivo deste artigo é mostrar como o uso de modelos de simulação pode proporcionar uma avaliação, em tempo de projeto, de alternativas arquiteturais para sistemas de monitoramento, detecção e notificação de quedas em ambientes domésticos baseados em IoT. Duas alternativas arquiteturais (uma convencional baseada em dispositivos vestíveis e uma que melhora a primeira e inclui sensores no ambiente do banheiro) são avaliadas. Dados reais de quedas foram utilizados durante as simulações. Resultados preliminares mostram um aumento de até 8,2\% na detecção de quedas da segunda arquitetura em relação à primeira.

\section{Introdução}

A população idosa vem crescendo gradativamente ao redor do mundo, o que acarreta uma crescente preocupação em como sanar os diversos problemas que assolam esse grupo, sendo a queda um desses. Estima-se que um a cada três adultos com mais de 65 anos experimentará uma queda por ano [Carey and Laffoy 2005], sendo a principal causa de consultas e internações médicas, gerando um gasto anual estimado em 10 
bilhões de dólares nos EUA e 581 milhões de libras no Reino Unido. Quedas representam a sexta principal causa de mortes em idosos acima de 65 anos, a segunda entre idosos de 65 a 75 anos, e a primeira para idosos acima de 75 anos [Carey and Laffoy 2005; Cotter et al. 2006].

Vários estudos na área de Internet das Coisas (do inglês Internet of Things - IoT) utilizam dispositivos de sensoriamento diversos como acelerômetros e giroscópios para o monitoramento de quedas [Schwickert et al. 2013]. Contudo, essas soluções requerem que o usuário acople um dispositivo justo ao seu corpo, normalmente ao tórax, cintura ou coxa [Ntanasis et al. 2017]. Isso implica que no momento que esses usuários estão no banheiro, eles não estão sendo monitorados para o caso de uma eventual queda. Além disso, as diferentes combinações de dispositivos trazem como consequência variações na precisão dos resultados na detecção de quedas.

Neste sentido, os autores acreditam que simulações podem ajudar a prever, ainda na fase de design de sistemas, o resultado obtido a partir de diferentes arquiteturas de sistemas IoT para monitoramento de quedas. Não foram encontradas, até o presente momento, pesquisas que utilizam simulações para abordar a queda de idosos em ambientes domiciliares.

Este artigo propõe avaliar um método tradicional de monitoramento de pacientes domiciliares, realizado por meio de acelerômetros, com a adição de um novo componente, onde o banheiro da residência é monitorado sem a necessidade de que um sensor seja vestido por um paciente. Esta pesquisa tem como hipótese que as abordagens tradicionais que utilizam um dispositivo acoplado ao corpo da pessoa atendem as necessidades de monitorar quedas quando a pessoa se encontra vestida. Contudo, em ambientes como o banheiro, onde há grande incidência de quedas de idosos, tal aparato pode causar desconforto ou não suprir as necessidades de monitoramento. A utilização de uma abordagem não invasiva, que não dependa do usuário, poderia identificar um maior número de ocorrências de quedas em comparação à abordagem tradicional. A pesquisa utiliza uma simulação para comparar o desempenho das duas arquiteturas de sistemas.

O artigo está estruturado da seguinte forma: a Seção 2 apresenta fundamentação teórica desta pesquisa; a Seção 3 descreve visões arquiteturais do sistema de monitoramento de quedas; a Seção 4 aborda o protocolo da simulação com cenário, objetivo, justificativa, métrica, instrumento de pesquisa e resultados; a Seção 5 relaciona trabalhos que envolvem monitoramento de quedas; e a Seção 6 discute as conclusões e as ameaças à validade deste estudo.

\section{Fundamentação teórica}

Uma queda pode ser definida como chegar ao chão ou a algum nível inferior de forma não intencional, sem que isso seja consequência de sofrer um golpe violento, perda da consciência, ou de ataque súbito de paralisia, como no derrame ou convulsão epiléptica [Gibson et al. 1987]. Dessa forma, qualquer evento que leve o objeto observado ao chão de maneira não intencional pode ser considerado como uma queda. Ao analisar os dados de um acelerômetro, pode-se identificar uma queda (de forma simplificada) como alterações na aceleração do objeto observado. De forma semelhante, o mesmo comportamento pode ser analisado por meio de outros sensores [Schwickert et al. 2013]. 
No monitoramento de quedas utilizando acelerômetros, um dispositivo deve ser carregado pelo objeto monitorado. O dispositivo analisa a aceleração de dois ou mais eixos, e mudanças súbitas nessa aceleração são identificadas como quedas [Abbate et al. 2010]. O monitoramento por radar doppler, por outro lado, ocorre ao anexar um dispositivo na parede ou teto de uma sala. O dispositivo emite ondas sonoras, e por meio do efeito doppler, identifica a aceleração de objetos na sala. Quando uma variação na aceleração ocorre, pode-se categorizar como uma queda [Mercuri et al. 2013].

A Internet das Coisas (IoT) permite que pessoas e coisas estejam conectadas a qualquer momento, em qualquer lugar, com qualquer coisa e qualquer um, idealmente usando qualquer caminho/rede e qualquer serviço [Perracini 2009]. Isso implica que, independente de onde o usuário esteja, aplicações IoT devem adequar-se ao contexto, utilizando sensores e atuadores para atingir esse objetivo.

Perera et al. [2014] sintetizam vários trabalhos de desenvolvimento de arquiteturas IoT em uma arquitetura em camadas, cada qual abordando uma problemática de IoT, descrita a seguir.

- Contexto de Aquisição: camada responsável sobre como e onde obter os dados que serão consumidos por uma aplicação.

- Contexto de Modelagem: deve fornecer diferentes técnicas de modelagem dos dados adquiridos de sensores, como ontologias, orientação a objetos, par chavevalor, dentre outras.

- Contexto de Interpretação: deve abordar um ou mais métodos de interpretação dos dados adquiridos e modelados, como raciocínio por ontologias, lógica de primeira ordem, lógica probabilística, aprendizado supervisionado, aprendizado não-supervisionado, etc.

- Contexto de Disseminação: camada responsável por definir como as informações adquiridas, modeladas e interpretadas chegarão às entidades interessadas e como essas mesmas informações serão visualizadas.

Esta organização arquitetural proposta por [Perera et al. 2014] influenciou as visões arquiteturais do sistema de monitoramento de quedas utilizado para a simulação, como visto na próxima seção.

\section{Visões arquiteturais do sistema-alvo}

Os modelos conceituais apresentados a seguir são uma representação em alto nível do sistema mencionado. A Estrutura de Entidades do Sistema é composta por entidades e aspectos. As entidades representam coisas que existem em um determinado domínio. Os aspectos descrevem um subsistema que compõe um sistema quando esse sistema é visualizado de uma determinada maneira [Zeigler et al. 2013], são rótulos para uma visão mais detalhada de uma entidade.

Nos modelos, as entidades são representadas pelo símbolo @e e os aspectos pelo símbolo 回. O sistema de monitoramento de quedas é composto por seis módulos, que serão descritos a seguir: aquisição, modelagem, interpretação, disseminação, armazenamento e emergência. Essa visão entidade-aspectos foi adotada por duas razões:

(a) ela representa a estrutura do sistema de software IoT e é utilizada para gerar a visualização do comportamento, que são as visões mínimas desejáveis no projeto de um sistema [Heuzeroth and Löwe 2003]; e 
(b) são modelos gerados automaticamente pelo framework de simulação $M S 4 M E$ (disponível em http://ms4systems.com/) como visualização dos modelos de simulação desenvolvidos e cuja execução é reportada na seção 5.

A camada de aquisição, descrita na Figura 1, é responsável por coletar os dados utilizados pelo sistema de monitoramento. Ela é composta por uma pessoa, um módulo de radar e uma veste de coleta de dados. A pessoa é responsável por gerar os dados do sistema. Suas atividades diárias são monitoradas pelo acelerômetro e pelo radar doppler. Tanto o radar quanto a veste de coleta capturam esses dados e os enviam para a camada de modelagem por meio do gateway.

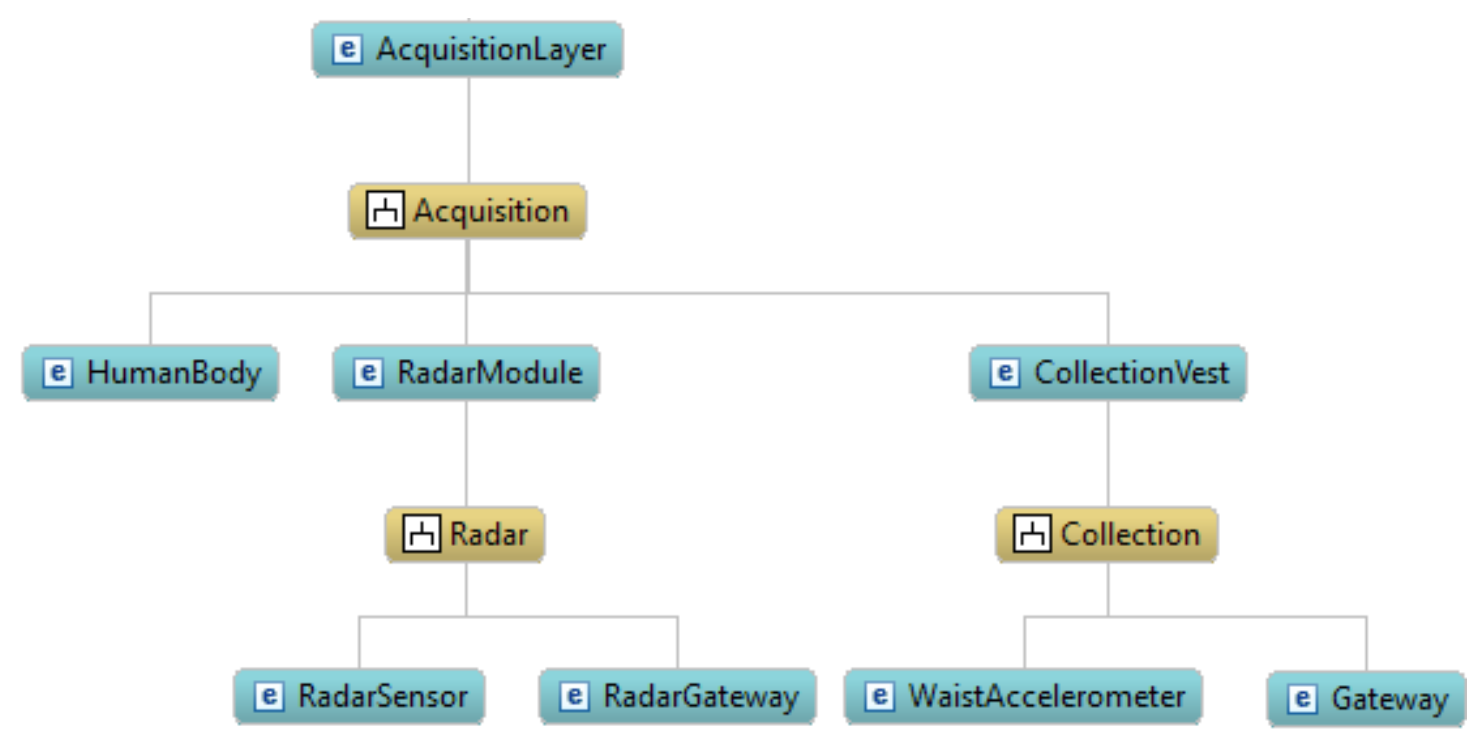

Figura 1. Modelo conceitual para a Camada de Aquisição.

A camada de modelagem é composta por um extrator de recursos. Em geral, sensores utilizados na IoT geram uma grande quantidade de dados. O extrator de recursos é responsável por organizá-los e definir quais informações serão enviadas para a camada de interpretação, ilustrada na Figura 2.

A camada de interpretação é composta por um avaliador de informações (i.e. DataEvaluation), responsável por identificar se uma queda foi registrada pelo acelerômetro ou pelo radar doppler. O resultado é enviado para a camada de disseminação, para que as partes interessadas sejam notificadas da queda, e para a camada de armazenamento, para que sejam armazenadas.

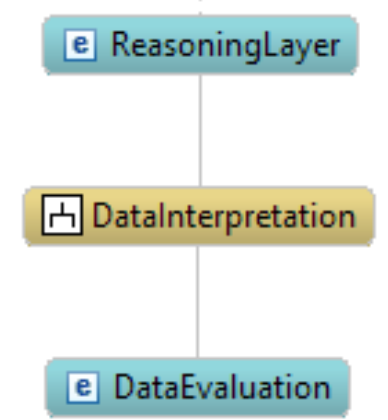

Figura 2. Modelo conceitual para a Camada de Interpretação. 


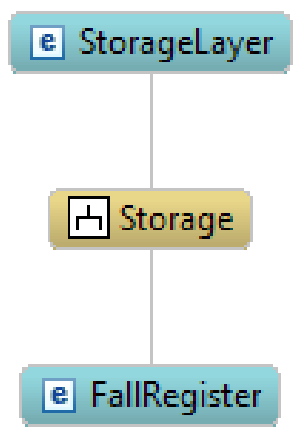

Figura 3. Modelo conceitual para a Camada de Armazenamento.

Representada na Figura 3, a camada de armazenamento armazena o histórico de quedas do paciente monitorado, possibilitando assim que aplicações tenham acesso ao quadro de quedas do mesmo. Por fim, a camada de disseminação, tal como ilustra a Figura 4, é composta pelo sistema de alerta e pelo cuidador. O sistema de alerta recebe o resultado enviado pela camada de interpretação e, caso uma queda tenha sido detectada, o sistema notifica o cuidador e o sistema de emergência.

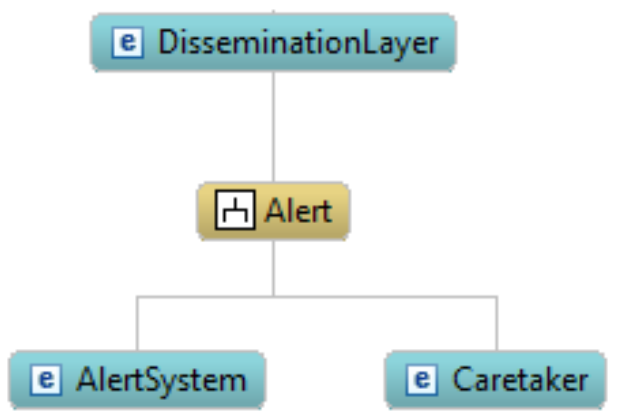

Figura 4. Modelo conceitual para a Camada de Disseminação.

A Figura 5 representa o sistema de emergência, composto por um centro de ajuda e uma ambulância. O centro de ajuda recebe o alerta da queda e envia uma ambulância para o local do acidente.

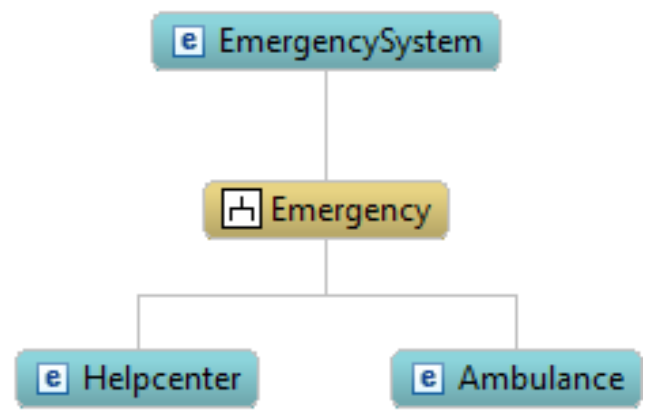

Figura 5. Modelo conceitual para o Sistema de Emergência.

Na próxima seção será apresentado o protocolo de pesquisa utilizado para realizar a simulação do sistema de monitoramento e detecção de quedas. 


\section{Protocolo da simulação}

O procedimento metodológico utilizado no experimento seguem as etapas definidas por [de França and Travassos 2016], nesta ordem: o planejamento da coleta de dados, a coleta e análise de evidências, e a discussão dos resultados.

Descrição do cenário. A simulação ocorre no contexto de um ambiente domiciliar, onde um idoso reside sozinho. A cidade onde ele vive é uma cidade inteligente e possui um sistema de emergência conectado. Um cuidador está à disposição de um idoso monitorado e, dependendo do estado de saúde deste, pode dirigir-se até a respectiva residência para auxiliá-lo.

O monitoramento é realizado por meio de dois dispositivos, como mostra a Figura 6. Em um cenário, há uma veste de coleta que deve ser utilizada pelo idoso com um acelerômetro na região da cintura para identificar quedas. O outro dispositivo é um emissor sonoro, instalado no banheiro da residência, que se baseia no efeito doppler para identificar a variação da aceleração de um corpo e, consequentemente, pode identificar a queda do paciente.

Objetivo. A simulação visa analisar a diferença da qualidade de detecção de quedas de duas arquiteturas de sistemas baseadas em IoT: a identificação de quedas de idosos por meio de acelerômetro e de radar doppler. Na primeira arquitetura, o monitoramento é feito de forma invasiva, sendo deficiente para a identificação de quedas quando o monitorado encontra-se despido. Já a segunda arquitetura realiza o monitoramento de forma não invasiva.

Justificativa. Tanto a arquitetura baseada em acelerômetros quanto a baseada em efeito doppler têm se provado útil para detecção de quedas. Contudo, restrições de usabilidade podem afetar a efetividade de tais dispositivos em alguns cenários. A simulação pode verificar, em tempo de design, a efetividade na deteç̧ão de quedas em um banheiro, onde a invasividade do dispositivo é de grande importância para o usuário.
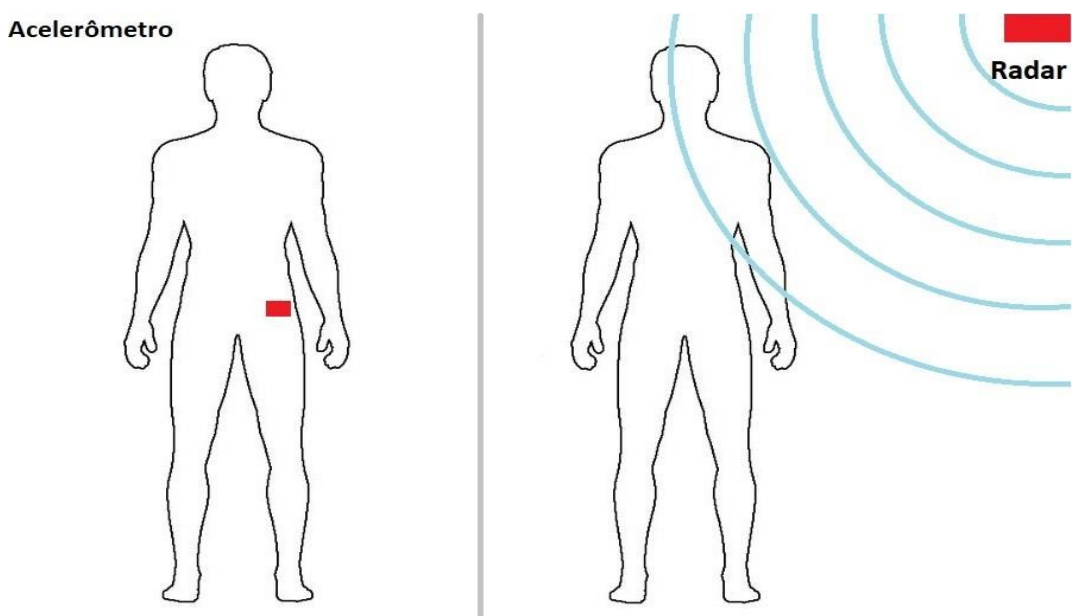

Figura 6. Monitoramento de paciente por meio de acelerômetro (à esquerda) e radar doppler (à direita).

Métricas. Aproximadamente $9 \%$ das quedas de idosos em um ambiente domiciliar ocorrem no banheiro, segundo [Wild et al. 1981]. Essa margem será aplicada em uma base de dados de quedas para identificar quantas dessas não seriam identificadas por uma arquitetura baseada na utilização de acelerômetros. 
Instrumentos de Pesquisa. O experimento foi realizado utilizando o formalismo DEVS (Discrete Event System Specification) [Zeigler et al. 2013] e a ferramenta MS4ME para desenvolver o modelo computacional e simular o comportamento de modo gráfico.

Modelo Computacional ${ }^{1}$. O modelo foi gerado automaticamente pela ferramenta MS4ME por meio das visões arquiteturais descritas na seção 3.

Condições de execução. A simulação foi executada em um laptop munido de processadori73517u @1.90GHz, 8GB RAM, SO Windows 10 versão 1903. A simulação teve duração de aproximadamente 4 horas.

Preparação dos dados. A simulação utiliza um dataset cujos dados já estão interpretados provenientes de um estudo anterior [Özdemir and Barshan 2014]. Os dados foram manipulados de forma que parte do dataset foi utilizado pelo acelerômetro, e outra parte do mesmo foi utilizado pelo radar doppler. O dataset inicial possui 16 mil estados - para esta pesquisa, ele foi dividido em 3 datasets menores, cada um contendo 5 mil estados (Os estados não utilizados foram descartados). A Tabela 1 apresenta um trecho dos dados e a respectiva estrutura do dataset utilizado.

Tabela 1. Trecho de dataset usado na simulação [Özdemir and Barshan 2014].

\begin{tabular}{|c|c|c|c|c|c|c|}
\hline Atividade & Tempo & Glicemia & Eletroencefalografia & $\begin{array}{c}\text { Pressão } \\
\text { sanguínea }\end{array}$ & $\begin{array}{c}\text { Frequência } \\
\text { cardíaca }\end{array}$ & $\begin{array}{c}\text { Circulação } \\
\text { sanguínea }\end{array}$ \\
\hline 4 & 7102,16 & 14148,8 & $-2381,15$ & 85 & 120 & 809 \\
\hline 5 & 7015,24 & 7336,79 & $-1699,8$ & 22 & 95 & 427 \\
\hline 3 & 8620,28 & 24949,9 & $-3198,06$ & 35 & 157 & 1519 \\
\hline 3 & 9238,73 & 39245,5 & $-2,59 E+03$ & 15 & 196 & 1885 \\
\hline 0 & 12276,4 & 59742 & -5101 & 56 & 249 & 2826 \\
\hline
\end{tabular}

Análise dos dados coletados. O dataset foi criado por 14 voluntários, que executaram um conjunto padronizado de movimentos, incluindo 20 quedas voluntárias e 16 atividades da vida diária, com uma média de 5 repetições. Isto resultou em um conjunto de dados com 2520 experimentos - cada linha na Tabela 1 corresponde a um período de 4 segundos. O dataset apresenta 6 estados possíveis para o paciente monitorado: parado (0), andando (1), sentado (2), caindo (3), cãibra (4) e correndo (5).

Resultados. De 5000 atividades analisadas, 748 quedas foram encontradas no primeiro grupo de dados. Dessas, 687 foram encontradas pelo acelerômetro e 61 quedas pelo radar doppler (8,2\% do total). Do segundo grupo, foram encontradas 153 quedas, sendo 139 via acelerômetro, e 14 por meio do radar doppler (9,2\% do total das quedas). Do último grupo de dados, foram encontradas 289 quedas: 266 pelo acelerômetro e 23 pelo radar doppler ( $8 \%$ do total das quedas).

Com uma média de 8,2\% das quedas (98 de 1190) identificadas pelo radar doppler, conclui-se que os resultados obtidos via simulação são condizentes com o estudo de [Wild et al. 1981], e que o uso de IoT com doppler no banheiro conseguiria identificar quedas que antes não seriam identificáveis.

1 A execução do modelo computacional pode ser visualizada por meio deste link. 


\section{Trabalhos relacionados}

Como afirmaram os autores, não foram encontrados trabalhos que utilizam simulações para abordar a queda de idosos em ambientes domiciliares. Entretanto, há pesquisas recentes que realizam experimentos com radares doppler para o monitoramento de quedas [Mercuri et al. 2013; Garripoli et al. 2013; Zhang and Lan 2018].

Em seus experimentos, Mercuri et al. [2013] atingem uma taxa de 94,3\% de assertividade na distinção entre quedas e atividades do dia-a-dia. Contudo, apenas quedas frontais foram analisadas e no ambiente de uma sala de estar. No estudo de [Garripoli et al. 2013], os experimentos demonstram uma taxa de 95\% de acertos, sem a ocorrência de falsos positivos. Em uma pesquisa mais recente, Zhang and Lan [2018] utilizam um radar doppler para identificação de quedas em um banheiro com uma assertividade de 73\% na identificação, sem a incidência de falsos positivos.

Demonstra-se que, em tempo de design de sistema e por meio de simulação, conseguimos identificar $100 \%$ das quedas representadas no dataset utilizado, enquanto que a melhor taxa de acerto encontrada nos trabalhos relacionados (que não utilizam simulação) é de 95 por cento.

\section{Conclusões}

Como alternativa ao método tradicional de monitoramento de quedas de idosos em domicílio com o uso exclusivo de acelerômetros, este artigo descreve uma solução arquitetural, baseada em simulação, com monitoramento baseado em acelerômetro e radar doppler. Essa solução é coerente com a necessidade de acompanhamento de idosos, mesmo em locais onde a privacidade e a invasividade do dispositivo afetam diretamente a relação do usuário com a aceitação da solução.

Ao compararmos com a literatura, o escopo do monitoramento apresentado pela simulação é maior; nas áreas não cobertas pelo radar doppler, o monitoramento é feito pelo acelerômetro. A assertividade na identificação das quedas pelo radar doppler é melhor; enquanto nos trabalhos relacionados a melhor taxa foi de 95\% [Garripoli et al. 2013], a simulação realizada neste trabalho conseguiu identificar todas as quedas. Mesmo utilizando apenas uma taxa de $9 \%$ do total das atividades do dataset, a simulação demonstrou que $8,2 \%$ das quedas seriam encontradas pelo radar doppler, um número significativo dada a natureza do local de monitoramento. Em um banheiro, em geral, há poucas superfícies acolchoadas e diversas quinas, o que tende a resultar em fraturas e internações, reforçando a relevância desta pesquisa [Schellenberg et al. 2019].

A utilização de simulação possibilitou a visualização dos componentes do sistema e suas interconexões, de forma que facilita a identificação de suas estruturas e comportamentos, bem como os ganhos trazidos por uma solução mista para o monitoramento de quedas.

Como trabalho futuro, considera-se a implementação de um algoritmo para identificação de quedas utilizando acelerômetros e de radares doppler, como passo anterior a se realizar experimentos em ambientes reais.

\section{Agradecimentos}

Renato Bulcão Neto agradece à CAPES/FAPEG pela bolsa de estudos de pós-doutorado (88887.305511/2018-00) feito no Dep. de Computação e Matemática da FFCLRP-USP. 


\section{Referências}

Abbate S.; Avvenuti M.; Corsini P.; Light J.; Vecchio A. (2010). “Monitoring of human movements for fall detection and activities recognition in elderly care using wireless sensor network: A survey”. In Wireless Sensor Networks, Chapter 9, IntechOpen.

Carey, D.; Laffoy, M. (2005). Hospitalisations due to falls in older persons. Irish Medical Journal, 98 (6):179-81.

Zhang, Y.W.D.; Lan, H. (2018). Bathroom fall detection based on ultrasonic Doppler effect. Journal of Shangai Normal University (Nature Sciences), 47(2):225-229.

de França, B.B.N.; Travassos, G.H. (2016). Experimentation with dynamic simulation models in software engineering: planning and reporting guidelines. Empirical Software Engineering, 21(3):1302-1345.

Wild, D.; Nayak, U.S; Isaacs, B. (1981). How dangerous are falls in old people at home?. British Medical Journal, 282(6260): 266-268.

Garripoli, C.; Mercuri, M.; Karsmakers, P.; Soh, P.J.; Pace, C.; Leroux, P; Schreurs, D. (2013). "Indoor fall detector based on Doppler radar". In Annual Symposium of the IEEE EMBS and IM Benelux Chapter, Brussels, Belgium, pp. 1.

Gibson, M.J.S.; Andres, R.O.; Kennedy, T.E.; Coppard, L.C. (1987). The prevention of falls in later life: a report of the Kellogg International Work Group on the Prevention of Falls by the Elderly. Copenhagen: Danish Medical Association.

Heuzeroth D., Löwe W. (2003). Understanding Architecture through Structure and Behavior Visualization. In: Zhang K. (eds) Software Visualization. The Springer International Series in Engineering and Computer Science, vol 734. Springer, Boston, MA.

Mercuri, M.; Soh, P.J.; Pandey, G.; Karsmakers, P.; Vandenbosch, G.A.E.; Leroux, P.; Schreurs, D. (2013). Analysis of an Indoor Biomedical Radar-Based System for Health Monitoring. IEEE Transactions on Microwave Theory and Techniques, 61(5):20612068.

Ntanasis P.; Pippa E.; Özdemir A.T.; Barshan B.; Megalooikonomou V. (2017). "Investigation of sensor placement for accurate fall detection". In 6th EAI International Conference on Wireless Mobile Communication and Healthcare, Milan, Italia, pp. 225232.

Özdemir, A.T.; Barshan, B. (2014). Detecting Falls with Wearable Sensors Using Machine Learning Techniques. Sensors, 14(6):10691-10708.

Cotter P.E.; Timmons S.; O'Connor, M.; Twomey, C.; O'Mahony, D. (2006). The financial implications of falls in older people for an acute hospital. In Irish Journal of Medical Science, 175(2):11-3.

Perera C.; Zaslavsky A.; Christen P.; Georgakopoulos D. (2014). Context aware computing for the internet of things: A survey. IEEE Communications Surveys Tutorials, 16(1):414-454.

Perracini, M.R. (2009). Desafios da prevenção e do manejo de quedas em idosos. BIS, Bol. Inst. Saúde (Impr.), (47):45-48. Disponível em $<$ http://periodicos.ses.sp.bvs.br/scielo.php?script=sci_arttext\&pid=S1518- 
18122009000200012\&lng=pt\&nrm=iso > . acesso em 14 jun. 2019.

Schellenberg, M.; Inaba, K.; Chen, J.; Bardes, J.M., Crow, E.; Lam, L.; Benjamin, E.; Demetriades, D. (2019). Falls in the Bathroom: A Mechanism of Injury for All Ages. Journal of Surgical Research, 234:283-286.

Schwickert, L.; Becker, C.; Lindemann, U.; Maréchal, C.; Bourke, A.; Chiari, L.; Helbostad, J.L.; Zijlstra, W.; Aminian, K.; Todd, C. (2013). Fall detection with bodyworn sensors: A systematic review. Zeitschrift für Gerontologie und Geriatrie, 46(8):706-19.

Zeigler, B.P.; Sarjoughian, H.S.; Duboz, R.; Soulíe, J.-C. (2013). Guide to modeling and simulation of systems of systems. Springer, 1st edition. 